\title{
约束线方法在生态学研究中的应用
}

\author{
郝蒿芳 ${ }^{1}$ 于德永 $^{1^{*}}$ 邬建国 ${ }^{1,2} \quad$ 郭勤峰 $^{3}$ 刘宇鹏 ${ }^{1}$ \\ ${ }^{1}$ 北京师范大学地表过程与资源生态国家重点实验室/人与环境系统可持续研究中心, 北京 $100875 ;{ }^{2}$ School of Life Sciences and Global Institute of \\ Sustainability, Arizona State University, Tempe, AZ 85287, USA; ${ }^{3}$ USDA FS, Eastern Forest Environmental Threat Assessment Center, 3041 Cornwallis Rd. \\ Research Triangle Park, NC 27709, USA
}

摘 要 生态系统格局和过程往往受到多个因子的共同影响, 故此反映两个生态学变量相互关系的散点图常常会表现为有 边界的散点云。基于数据均值或中值分析的传统统计方法不适用于散点云数据的分析。散点云所表征的不是两变量之间的相 关关系, 而是限制作用关系。约束线(包络)方法为提取散点云边界、理解限制变量对响应变量的作用, 以及预测响应变量的 潜在最大值提供了有效手段。该文对应用约束线方法研究生态学问题所取得的成果进行总结与归纳, 介绍了约束线概念的发 展历程、提取方法, 从物种分布、种群行为及作物产量优化三个方面总结了约束线方法的优点及适用性，概述了当前约束线 方法应用研究面临的问题与挑战, 指出约束线方法应结合其他统计方法, 实现对生态过程的准确理解, 此外, 还应重视约束 线方法的尺度依赖性。最后，该文展望了约束线方法在研究生态系统服务关系和土地系统优化等方面的应用前景。

关键词 散点云; 有效边界; 约束包络; 限制响应; 物种分布; 种群行为; 优化

引用格式: 郝莣芳, 于德永, 鸟建国, 郭勤峰, 刘宇鹏 (2016). 约束线方法在生态学研究中的应用. 植物生态学报, 40, 1100-1109. doi: 10.17521/cjpe. 2016.0152

\section{Constraint line methods and the applications in ecology}

\author{
HAO Rui-Fang ${ }^{1}$, YU De-Yong ${ }^{1 *}$, WU Jian-Guo ${ }^{1,2}$, GUO Qin-Feng ${ }^{3}$, and LIU Yu-Peng ${ }^{1}$ \\ ${ }^{1}$ Center for Human and Environment System Sustainability (CHESS), State Key Laboratory of Earth Surface Processes and Resource Ecology (ESPRE), Bei- \\ jing Normal University, Beijing 100875, China; ${ }^{2}$ School of Life Sciences and Global Institute of Sustainability, Arizona State University, Tempe, AZ 85287, \\ USA; and ${ }^{3}$ USDA FS, Eastern Forest Environmental Threat Assessment Center, 3041 Cornwallis Rd. Research Triangle Park, NC 27709, USA
}

\section{Abstract}

With increasing data availability in the big data era, many traditional statistical analyses based on the mean or median are insufficient or inappropriate to elucidate the complex patterns of variation. This is particularly the case when multiple factors are involved and the bivariate scatter occurs as scatter clouds. In such circumstances, constraint line (or envelope) method could be an alternative and effective tool to extract the data boundaries, thus improves our understanding of the complex relationships between limiting factor and response factor. Here, we synthesize the major findings and achievements in the field of applying the constraint line method in ecology. Specifically, we first describe the history and development of the constraint line method. We then discuss the techniques to establish the constraint lines with examples, and discuss the applications and implications of the constraint lines in species distribution, population performance, and optimization problem. We suggest simultaneously application of both constraint lines and regression techniques to the same datasets to achieve a comprehensive understanding of ecological process and underlying mechanisms. Such combined methods should be used with special attention to the role of spatial heterogeneity and scale dependency. We also discuss in detail the potential applicability of the constraint line method in studying the linkages between ecosystem services, and land system design.

Key words scatter cloud; informative boundary; constraint envelope; limiting response; species distribution; population performance; optimization

Citation: Hao RF, Yu DY, Wu JG, Guo QF, Liu YP (2016). Constraint line methods and the applications in ecology. Chinese Journal of Plant Ecology, 40, 1100-1109. doi: 10.17521/cjpe.2016.0152

收稿日期Received: 2016-04-25 接受日期Accepted: 2016-07-23

* 通信作者Author for correspondence (E-mail: dyyucas@163.com) 
在我们研究两个与复杂生态过程有关的变量之 间的相互关系时, 最常用的方法是控制变量法, 即 假定其他因子不变并对两变量不造成约束, 进而采 用回归分析或相关分析。然而这一假设在复杂生态 过程中常常难以满足, 一方面, 生态学相关研究往 往以野外采样数据为基础, 很难控制其他因子处于 理想状态; 另一方面, 在野外采样过程中, 研究者 一般不对其他因子进行测量记录, 因此在分析两变 量之间关系时, 有时难以得到正确的结论(Cade et al., 1999; Cade \& Guo, 2000)。除此之外, 野外调查 数据的分布常常呈现出散点云 (scatter cloud) (Thomson et al., 1996)的形态。在这种情况下, 我们 更关注响应变量在测量因子的限制作用下, 散点云 边界的变化率(Thomson et al., 1996)。约束线(包络) 分析是可以准确刻画数据边界规律、寻找变量之间 限制作用的有效方法。

\section{1 什么叫约束线方法}

约束线的概念最早由Webb (1972)提出, 当时约 束线被称为边界线(boundary line)。Webb (1972)认为 约束线出现在有因果关系的两个变量间, 落在数据 的边界, 代表种群生长的最好状态(Webb, 1972)。因 此, 约束线最早在生态学中的应用, 主要指限制因 子(limiting factor)将响应因子(response factor)约束 在一个范围内。此时, 约束线强调边界性(boundary) 和因果关系(causality)。Schnug等(1996)沿用边界线 的概念, 认为它对应散点云的上边界(upper edge)。

Blackburn等(1992)发现, 由于种内竞争, 物种多度 (abundance)与生物体大小的约束关系呈现斜率为 -0.75 的直线, 与自疏法则 (self-thinning)非常吻合, Blackburn等(1992)将约束线称为散点上边界(upper bound)。在Blackburn等(1992)的理论基础上, Thomson等(1996)将约束线方法应用到物种空间分布的 研究中, 并认为植物的空间分布与多种环境因子有 关, 数据点广泛地分布在某一个限制范围内, 表现 为有信息的点云(informative point cloud), 称这种现 象为因子天花板分布(factor-ceiling distribution)。直 到 1998 年, Guo 等 (1998) 整合 “boundary line” 与 “factor-ceiling”的概念, 在研究种群分布(响应变量) 与环境因子(限制变量)的关系时发现, 数据分布的 边界线可以排除其他因子对响应变量的影响, 能够 更好地代表限制变量与响应变量之间的关系, 响应 变量的分布一般不超越“constraint line”, 即约束线。 Mills等(2009)与Medinski等(2010)沿用Webb (1972) 提出的“boundary line”的概念, 在研究土壤属性对 植物丰富度(species richness)的影响时指出, 随着某 种土壤属性的梯度变化, 植物丰富度分布在某一数 值范围内, 称为生态位(niche), 约束线代表植物丰 富度能够达到的最大值。

在复杂的生态过程中, 两个变量之间除相互作 用外, 还可能受到其他很多因素的影响, 进而这两 个变量的关系往往呈现类似散点云的分布特征 (Blackburn et al., 1992; Thomson et al., 1996; Pittman \&Turnblom, 2003)。之所以会出现这种现象, 是因为 限制变量不能完全控制响应变量的变化, 而是对响 应变量有限制作用, 从而使响应变量的分布不能超 过某个范围(Thomson et al., 1996)。传统的回归分析 和相关分析并不适用于此类呈散点云分布的两个变 量之间相互关系的研究, 因为传统的统计方法着眼 于变量均值的关系, 要求数据分布在均值周围, 而 散点云并不是围绕某一均值或中值线分布, 这与回 归分析和相关分析的基本假设相违背。尽管多元回 归能够分析多个自变量对因变量的贡献, 但很难从 生态学机理上进行解释。相比于传统的线性回归和 相关分析方法, 约束线方法能够更好地刻画由于多 因素影响的复杂生态系统中限制变量对响应变量的 限制作用(Guo et al., 1998)。

如图1所示，散点云的不同部分能够代表不同 的生态过程(Cade \& Noon, 2003)。在理想情况下, 生 物体的响应主要受到测量因子的作用, 其他的潜在 影响因子都处在自由水平, 即不会对生物体造成限 制作用(图1A)。例如, 假设某种生物的生长速率由 单一限制因子(食物密度)决定, 且两者呈现线性关 系, 如果其他所有因子 (比如温度)的潜在影响是最 小的或者并未达到能够对生物体生长起到限制作用 的水平, 那么生物的生长速率与食物密度将会围绕 某条直线分布，采用简单的线性回归或者相关分析 就能够得到两者之间的关系。但是, 在现实采样过 程中, 除食物密度之外的其他因子(比如温度)也会 对生物的生长速率造成影响, 那么这些点将会分布 在直线下(图1B、1C、1D)。在这种情况下，如果仍 然用相关分析, 得到的结果可能显示生物生长速率 和食物密度之间并无关系, 然而, 如果采用约束线 方法, 即使采样点数据受到其他因子的影响, 散点 
$\mathbf{A}$, 理想状态: 只有测量因子有限制作用

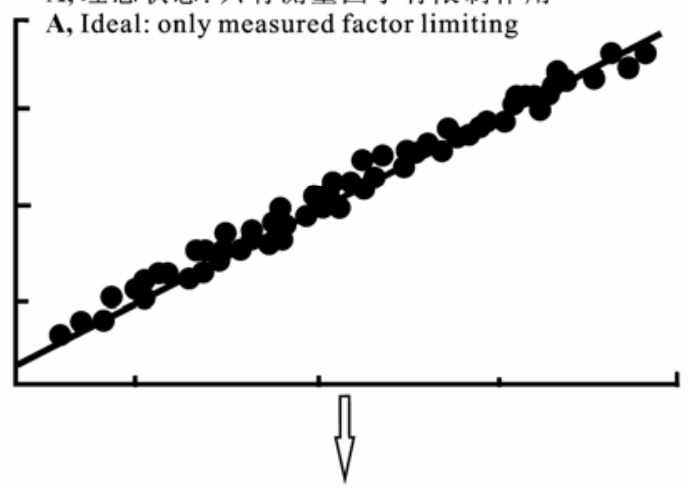

B, 未测量的一个因子在一些地方有限制作用 B, Unmeasured factor limiting at some sites

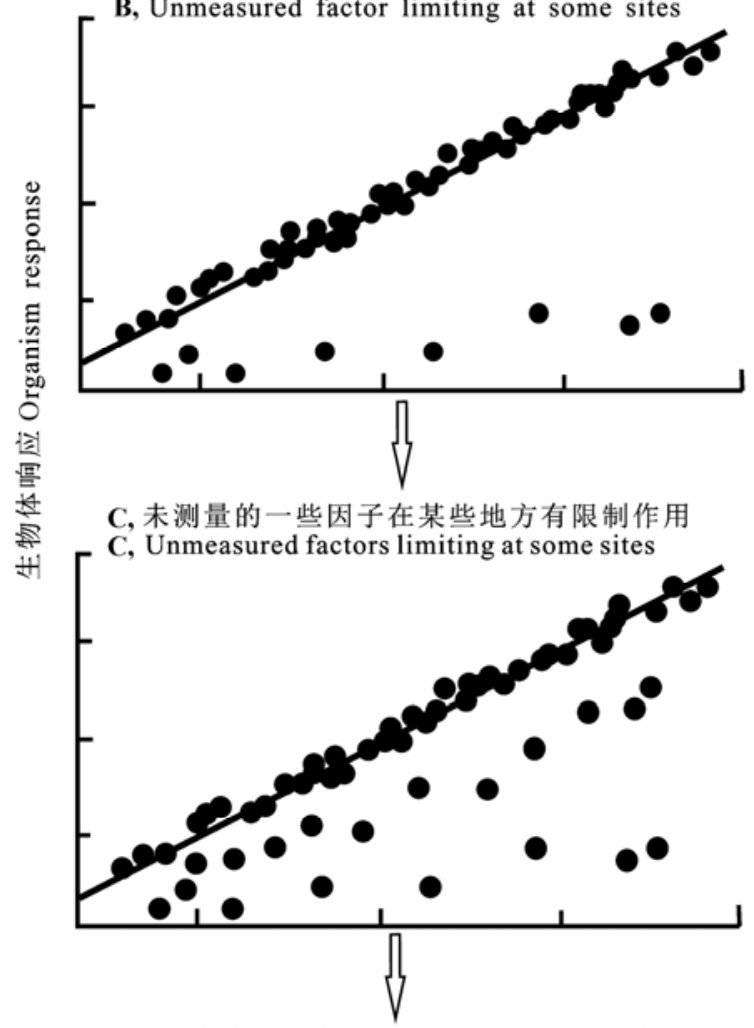

D, 未测量的一些因子在许多地方有限制作用

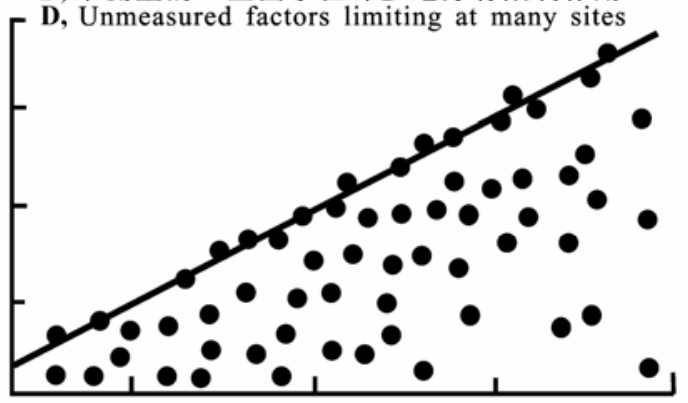

测量因子 Measured factor

图1 理想状态和现实情况下测量因子与生物体响应的散 点分布图(修改自Cade和Noon (2003))。

Fig. 1 The scatter diagrams showing the relationships between an independent factor and organism response under the ideal (top: single causality) and more realistic situations (bottom: multicausality) (Adopted from Cade \& Noon (2003)).
的上边界仍然能够表征生物的生长速率与食物密度 之间的关系(Cade \& Noon, 2003)。因此，如果数据分 布呈现散点云特征时, 约束线方法是表征两个变量 关系的较好选择(Guo et al., 1998; Cade \& Noon, 2003), 约束线表征响应变量在限制变量作用下的 分布范围或者能够达到的潜在最大值(Kaiser et al., 1994)。

\section{2 约束线的绘制方法}

最初, 约束线是通过视觉观察散点云的分布形 态人为绘制(Scharf et al., 1998)。随着相关研究越来 越多, 约束线绘制的方法也逐渐增多, 并趋于成熟。 目前, 主要有以下4种绘制方法: 参数法、散点云网 格法、分位数回归法和分位数分割法。

(1)参数法(parameter method)。如基于Yoda自疏 法则, 植株生物量大小与种群密度之间的关系已经 相对明确, 只需要针对不同的研究群体确定自疏法 则中的参数即可得到约束线(Guo et al., 1998; Lessin et al., 2001)。这种方法的优点是, 以生态学理论为 基础, 能够对生态过程的动态进行全面解析, 其缺 点是应用范围较小。

(2)散点云网格法(dividing scatter cloud into size classes)。首先将散点云的限制变量与响应变量划分 为若干区间, 进而将散点云划分为网格, 通过计算 每个网格中数据点的特征值(包括最大值、最小值、 平均值、密度等)来确定边界点的位置, 最后对边界 点拟合得到约束线。在此类方法中, 网格的划分又 包括根据点密度划分和点数值大小划分两种。这种 方法的优点是能够实现约束线的完美展示, 缺点是 网格的划分较为主观, 不同的划分数目对结果有较 大影响。Blackburn等(1992)最早将限制变量每一区 间中点数最多的网格作为约束线边界网格, 进而提 取约束线。Thomson等(1996)将限制变量按照数值大 小等分为若干区间, 取每个区间中响应变量的最大 值作为边界点, 称这种方法为逻辑切片法(logistic slices)。Roberts和Angermeier (2007)也采用类似的方 法, 用 0,1 来表征物种出现和不出现两种状态, 将观 测数据分为 5 个区间, 并保证所有区间中采样点的 数量相同, 将每一区间中最大的两个观测值取平均 值作为边界点, 最后使用逻辑斯蒂克(logistic)回归 得到约束线。

(3)分位数回归法(quantile regression)。这种方法 
以加权最小一乘法为算法, 以不同的分位数为基准, 设法使各数据点到回归线的纵向距离的绝对值之和 为最小, 这种方法应用较为普遍, 但是它的缺点是 对曲线或者非线性的分布形态不能较好地表征 (Cade et al., 1999; Lessin et al., 2001; Coomes \& Allen, 2007; Strong, 2011)。Anderson和Jetz (2005)采用 极端分位数回归绘制恒温动物能量支出边界线。

Chassot等(2010)通过连续取不同的分位数理解海洋 初级生产力对渔业的限制作用。Cade和Noon (2003) 以分位数回归为基础总结了约束线方法在生态学问 题研究中的应用, 并指出不同分位数得到的结果对 理解生态过程有重要意义。

(4)分位数分割法(segmented quantile approach)。 Medinski (2007)和Medinski等(2010)将逻辑切片法 (logistic slices)与分位数回归相结合, 首先将限制变 量按照数值大小分区, 取每个区间中所有点的 $95 \%$ 分位数和 $10 \%$ 分位数分别作为上下边界点, 最后对 上边界点进行拟合得到约束线, 他称这种方法为分 位数分割法。在此基础上, Mills等(2009)采用分位数 分割法获得边界点, 对边界点进行最小二乘法拟合, 从直线、二次函数、对数函数、指数函数四类函数 中取拟合决定系数最大的类型作为约束线的拟合结 果, 相比前3种方法, 这种方法更具有统计学基础。

\section{3 约束线方法在分析生态学问题中的应用}

目前, 约束线方法主要用于分析物种分布、物 种行为对环境因子的响应和作物产量优化 3 个方面。 在物种分布相关研究中, 研究者往往以自疏法则为 基础, 使用约束线定量刻画生态系统结构及生物个 体动态生长过程中影响物种分布的主要生态过程 (Guo et al., 1998; Austin, 2007); 在物种行为对环境 因子响应的研究中, 往往以散点云的信息边界为基 础, 只关注散点云的约束线, 而不在意边界内部采 样点的变化趋势(Blackburn et al., 1992; Horning, 2012; Schneider et al., 2012); 在作物产量优化问题 的研究中, 限制变量对作物产量的影响具有空间异 质性和尺度效应, 约束线方法得到的结果只代表作 物产量在某个限制变量作用下能够达到的最大值 (Medinski, 2007; Wang et al., 2015a)。

\section{1 约束线方法用于研究种内竞争对物种分布的 影响}

Blackburn等(1992)研究动物个体大小与分布密
度之间的关系，采用约束线方法与最小二乘法进行 对比分析, 他们认为虽然自然资源因子不能单独决 定物种的多度, 但仍能决定物种在某个资源状态下 可以达到的最大多度。研究人员选取一系列靠近约 束线的数据点, 发现约束线的斜率趋近于 -0.75 , 符 合Yoda自疏法则。在此基础上, Guo等(1998)用约束 线方法研究沙漠植物种群密度与植株生物量之间的 关系, 以及由于种内竞争而导致的植物死亡率。由 于植物生长过程受到许多生物和非生物因素的影 响, 引起植株个体大小呈现较大的差异, 根据自疏 法则, 植株个体大小与植株密度之间应该呈现一个 上边界。如图2所示, 上边界表征自疏线, 种内竞争 非常激烈, 箭头线表征植株生长过程的轨迹, 1,2 代 表不同种群密度情形。在无种内竞争的环境下, 植 株生长沿着 $\mathrm{R}_{1}$ 轨迹进行, 直到植株大小到达自疏线, 此时, 生长资源(空间、养分等)受到限制, 种内竞争 发生。在自疏线上, 一些植株的生长一定要以其他 植株的死亡为代价, 那么, 植株个体生长沿着 $\mathrm{R}_{2}$ 轨 迹进行。在整个过程中, 种群 2 由于种内竞争植株死 亡数最多, 而种群 1 并没有经历自疏过程。同样, Lessin等(2001)使用散点云上边界的约束线定量研 究沙漠一年生植物种群的竞争行为, 他们将所有个 体数据作为研究对象, 采用回归分析发现沙漠一年 生植物大小和种群密度与其邻近种群地上生物量均 无关, 但是, 邻近种群地上生物量却对目标种群植 株大小的潜在最大值有显著影响。Guo和Rundeal (1998)结合回归分析与约束线方法研究火烧从林在 演替中的自疏过程, 发现自疏过程随着物种生物量 的累积越来越明显。以Yoda自疏法则为基础, 研究 种内竞争引起的物种分布规律成为约束线方法的主 要应用之一(Cade \& Guo, 2000; Guo et al., 2000)。

\section{2 约束线方法用于研究种群行为对环境因子的 响应}

表征环境因子对物种多度与生物体大小的限制 作用是约束线方法的另一主要应用(Nishihiro et al., 2000; Jansen et al., 2007; Strong, 2011), 其中, 环境 因子包括生物因子与非生物因子。Thomson等(1996) 采用约束线方法分析了百合(Lilium)开花植株、百合 幼苗、地鼠和岩石分布之间的关系, 揭示了幼苗密 度与开花植株密度之间负向关系的边界特征, 所有 散点的分布形成一个三角形, 较多的幼苗只会出现 在开花较为稀疏的地方, 此外, 研究人员引入影响 


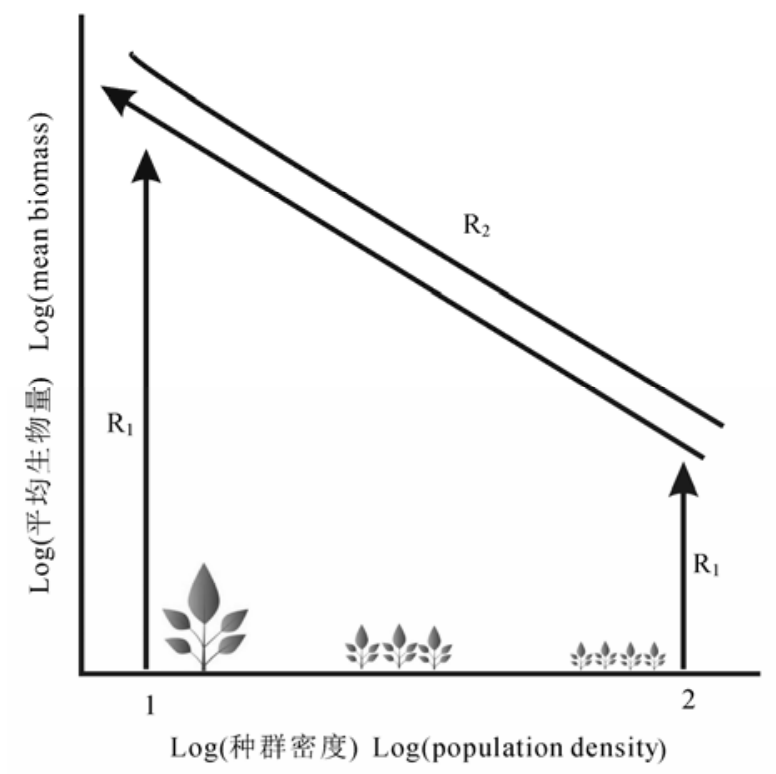

图2 邻近种群密度与生物量之间的异速自疏关系, 横坐 标为种群密度的对数, 纵坐标为种群平均生物量的对数(修 改自Guo等(1998))。R, 轨迹。

Fig. 2 The allometric thinning relationship between the neighborhood population density and the average biomass (Modified from Guo et al. (1998)). R, trajectory.

幼苗和开花植株的其他干扰因子, 包括地鼠和岩石 等, 通过路径分析验证了幼苗密度和开花密度之间 存在限制关系。Clark和Clark (1999)分析了热带雨林 9 种植物在不同树龄时期的生长能力, 发现用材林 树种即使在老龄期仍然有较强的生长能力, 树龄对 树木利用资源条件生长的限制作用不明显。Strong 等(2011)采用约束线方法发现随着白云杉(Picea glauca)冠层盖度的增大, 林下不同物种最大多度均 表现为下降趋势。气象要素作为限制因子对植株影 响的相关研究也屡见不鲜, 例如, Austin (2007)在研 究北非和中东地区归一化植被指数 (NDVI)与降雨的 关系时发现, 随着降雨量增多, 物种最大多度呈单 峰形式变化。Jansen等(2007)通过研究大范围植被对 温度季节周期变化的适应能力, 发现植物代谢率在 冬季与春季达到最大, 而在夏季与秋季常常有所降 低。为了将生物因子与非生物因子的影响剥离开, Coomes和Allen (2007)选择约束线方法研究光照和 营养物质对植物生长的限制作用时发现, 光照对幼 小植株的生长有较大影响, 而营养物质对不同年龄 植株的生长都有影响, 同时, 植株生长速率随着纬 度的增加而降低。

除陆生植物外, 约束线方法在研究水生植物对
环境因子的响应方面也得到了广泛应用, Krause等 (2000)发现大叶藻(Zostera marina)的生长速率随着 水深呈现指数型下降趋势。Schröder等(2005)采用非 线性分位数回归方法分析了沼泽植物多度对环境因 子的响应，种间竞争、资源贵乏、物种灭绝等原因 都可能造成沼泽植物物种多度降低, 所以基于均值 或者中值的传统研究方法会低估环境因子对沼泽植 被的影响。沼泽植物物种分布与土壤有机质含量之 间的关系可能是由于沼泽植物获取营养物质量和土 壤通风性决定的, 并不是土壤有机质对植物的直接 影响。

约束线方法还应用于研究动物行为对环境因子 的响应。Scharf等(1998)发现猎物大小与捕食者大小 之间的关系呈现多边形分布, 有较为明显的约束包 络(constraint envelope)特征。Roberts和Angermeier (2007)分析了 3种鱼在河流中的分布, 发现鱼类的活 动范围受到季节、河流流量、温度以及鱼类种类、 年龄、性别等多种因素的影响, 约束线方法能够准 确分析多种因子对鱼类活动范围的限制作用。

Anderson和Jetz (2005)研究了限制恒温动物能量支 出的主要因子, 发现恒温动物能量支出较高时, 能 量支出限制要素为生理因子, 能量支出较低时, 能 量支出限制要素为外界环境因子。

除自然环境因子的限制作用外，人类活动干扰 对种群行为的限制作用更为强烈, Wang等(2015b)发 现人类放牧活动对东北虎多度的限制作用呈现对数 型增大趋势。Walsh等(2005)在总结城市综合特征时 指出, 随着城市化进程的加剧, 不透水面百分比对 生物生存环境的限制作用表现为三种形态: 直线加 剧型、“S”型以及对数型。Wang等(2001)选择47个流 域为研究区, 发现随着流域内城市不透水面百分比 的增加, 鱼类多样性的最大值呈指数型下降趋势。

采用约束线方法研究种群行为对环境因子的响 应涉及较多因素, 与基于两个变量之间的限制作用 和自疏法则得到的约束线含义有较大区别。由自疏法 则得到的约束线能够排除其他因子的作用，只关注 由种内竞争引起种群个体死亡的规律, 而基于种群 行为对环境因子响应得到的约束线只能表征在测量 因子的限制作用下, 种群行为响应的变化范围或最 大值, 并不能排除测量因子之外的其他因子的影响。

\section{3 约束线方法用于作物产量优化}

Webb (1972)最早将约束线方法用于研究作物 
产量优化, 通过分析影响作物产量的限制因子, 可 以合理配置作物类型、优化管理措施。Evanylo和 Sumner (1987)使用约束线方法寻找大豆(Glycine $m a x)$ 生产过程中的土壤养分最佳值, 土壤养分最佳 值指在某种条件下，使作物产量达到最大值时对应 的土壤养分含量, 当土壤养分少于最佳值时会限制 作物产量。根据作物平均产量计算的土壤养分最佳 值可能低于真实值, 而约束线方法能够根据作物最 大产量计算土壤养分最佳值。例如, 在图3中, 1、2 为两种不同的情况, 如果 $x$ 变量在 $a$ 点, 对应的作物 产量不能超过 $a^{\prime}$, 可以是比 $a^{\prime}$ 低的任何值。在情况 1 下, $x$ 变量的限制作用最强, 它的变化可能会引起作 物产量的响应。在情况 2 下, 当变量 $x$ 为 $b$ 值时, 能够 得到作物产量的最大值, 但并不是总能获得最大值, 因为一些其他因子可能会限制产量, 从而使作物产 量分布在 $b^{\prime}$ 之下。所以, 当 $x$ 变量在最佳值时, 任何 大小的作物产量都能得到, $x$ 变量对作物产量不起限 制作用。

用约束线方法分析土壤属性对作物产量和植物 丰富度影响的相关研究也越来越多(Medinski, 2007; Mills et al., 2009; Medinski et al., 2010; Wairegi et al., 2010)。Medinski (2007)采用约束线方法将土壤 渗透能力、黏砂含量、土壤电导率以及土壤酸碱度 等土壤属性作为限制变量, 以不同生长期的植物物

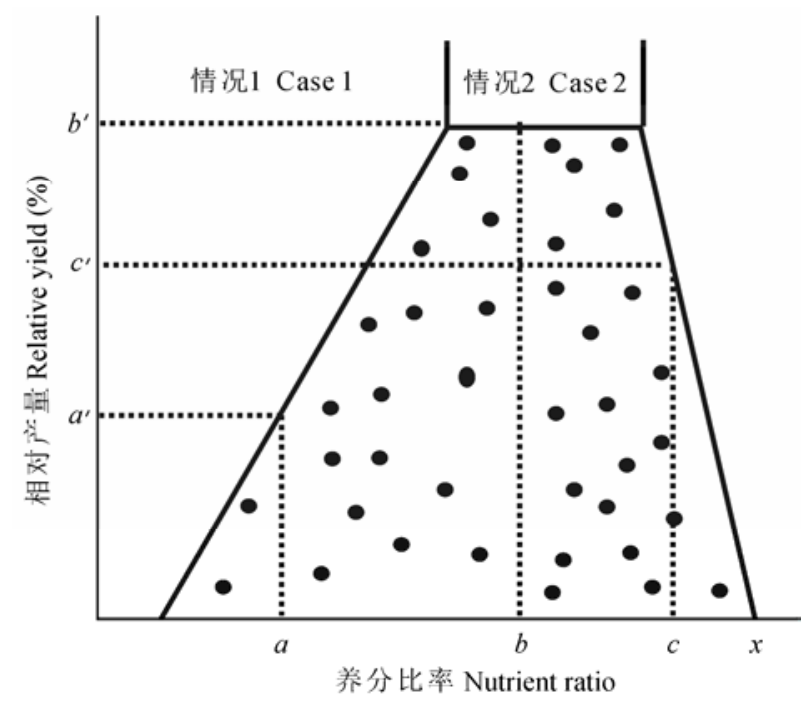

图3 植物组织的养分比率与某种作物相对产量的关系(修 改自Sumner (1978))。

Fig. 3 Relationship between relative yield of a crop and the value of a nutrient ratio in a given tissue (Modified from Sumner (1978)).
种丰富度为响应变量, 分析了每一种限制变量对物 种丰富度的约束关系, 约束线表示响应变量的潜在 最大值或是可预测的限制点, 植物丰富度的最大值 应该出现在资源条件刚好受到限制，除测量因子之 外的其他影响因子并未达到限制水平时, 此时, 并 不是所有植株都能够存活, 这与Guo等(1998)在研 究种内竞争时对约束线的解释类似。Wairegi等 (2010)研究东非高地香蕉(Musa paradisiaca)种植环 境时发现, 当降雨量从 $800 \mathrm{~mm}$ 增加至1 $200 \mathrm{~mm}$ 时, 降雨对香蕉产量的限制作用逐渐减弱直至消失, 整 个变化过程曲线呈对数形式。Medinski等(2010)认为 约束线方法所表示的响应变量的最大值并不能保证 其不受其他因子的影响, 只表示响应变量不受其他 因子的限制作用。因此, 在约束线上, 响应变量受到 限制变量的限制作用最强, 其他因子可能对响应变 量有影响, 但并不是决定响应变量变化的限制因子, 约束线下的点则表示响应变量受到其他因子的限制 作用。Mills等(2009)认为约束线方法虽然能够有效 识别两个变量之间的关系, 但是这并不意味着响应 变量的分布只受某一个因子的影响, 比如, 在土壤 渗透能力较低时, 点地梅(Eragrostis lehmanniana) 的植被盖度较小, 但是这并不意味着这种植物不能 忍受极端干旱的土壤, 可能是其他因子(如土壤通 气性或者土壤养分含量)对植被盖度产生了影响。

Huston (1999)认为约束线方法得到的结果具有 尺度依赖性。Wang等(2015a)研究能够提高咖啡 (Coffea linn)产量的重要生物、非生物因子时发现, 在咖啡生长的不同阶段, 主要的限制变量不同, 而 且这种限制作用是有空间异质性的, 所以, 咖啡产 量对某一个限制变量的响应可能会由于其他因子的 变化而发生改变。Robertson等(2015)采用约束线方 法预测新西兰河口物种对泥沙沉降增加的响应, 根 据种群密度最大值计算得到了最优的泥沙分布范 围, 从而为优化当地生态系统管理提供了有效的建 议。

\section{4 讨论}

\section{1 约束线方法在生态学研究中面临的问题与挑战}

自 1972 年边界线 (boundary line) 提出 (Webb, 1972) 以来, 约束线方法在生态学中的应用越来越 多, 目前国内相关研究较少。约束线方法能够弥补 传统统计方法在解译散点云数据分布时的缺陷, 有 
助于理解复杂生态过程中重要因子的相互作用机 理。但是，约束线方法还存在一些问题与挑战。

(1)尽管约束线方法旨在分析两个变量之间的 限制作用, 但是很多研究发现, 约束线所表征的关 系, 可能并不是由于限制变量对响应变量的直接作 用引起, 而是受到其他因子的影响 (Mills et al., 2009)。所以, 约束线方法结合其他统计方法, 如通 径分析、因果检验等, 有助于实现对生态过程的准 确理解。

(2)散点云约束包络由多条约束线组成, 约束包 络的大小、形状和它在多维空间中的位置蕴含着重 要的生态学信息, 应结合约束线、约束包络与回归 分析全面理解生态学变量之间的关系以及相关生态 学机理。

(3)约束线方法具有空间尺度依赖性(Huston, 1999), 但是这方面的研究有待深入。在使用约束线 方法时, 只有根据研究目的选取合适的研究尺度才 能深入理解限制变量与响应变量的关系。

(4)在绘制约束线时, 我们期望尽可能包含所有 数据点, 但是, 采样数据中常常会存在异常点, 如 何去除异常点还未有完善的方法, 需要根据具体的 研究问题, 甄别各种约束线绘制方法的准确性。

(5)以大量观察和测量数据为基础的约束线方 法受到数据可获取性的限制。然而, 在新兴学科和 “大数据时代”下迅速增长的样本量能够促进约束线 方法的使用。

\section{2 约束线方法在生态学问题研究中的应用前景}

约束线方法为我们研究复杂生态过程中限制变 量与响应变量之间的关系提供了新思路。不论其生 态学基础还是技术提取层面, 已有研究为约束线方 法的发展奠定了坚实基础, 相信约束线方法在未来 的生态学和相关学科交叉研究中会得到广泛应用。 基于对约束线方法的理解, 本文从生态系统服务关 系和土地系统设计两方面讨论约束线方法的潜在适 用性。

生态系统服务指人类从自然生态系统获取的利 益(Alcamo et al., 2003), 是满足和提高人类福祉的 必要物质基础(Wu，2013)。理解生态系统服务之间 的关系是实现生态系统服务有效管理的前提条件。 目前, 生态系统服务之间的关系分为权衡、协同和 无关三类(Bennett et al., 2009)。权衡指一种服务的 提高以另一种服务的降低为代价, 协同指两种或多
种生态系统服务同时增加或减少的现象(Bennett et al., 2009)。相关分析是研究生态系统服务关系普遍 采用的一种方法, 两种服务呈现正相关则认为是协 同关系, 负相关则为权衡关系(Raudsepp-Hearne et al., 2010)。但是, 如果采样点较多, 从生态系统服务 关系散点图中可以发现，所有散点可能并不是围绕 某一条线分布, 而是趋向于分布在某一条线下 (Raudsepp-Hearne et al., 2010)。此外, 生态系统服务 关系除受它们之间相互作用影响外, 还受很多其他 因子, 如气象因子、地形因子、地表覆盖因子等的 影响。这些因子的复杂作用也使得生态系统服务之 间并非呈现简单的线性关系。约束线方法为理解生 态系统服务之间的关系以及影响生态系统服务关系 的相关生态过程提供了一条新思路。约束线与帕累 托效率有相似的含义(Thomson et al., 1996), 目前在 生态系统服务关系的研究中, 也有一些研究以帕累 托效率为依托, 用边际函数表征生态系统服务之间 的权衡关系(Lautenbach et al., 2010)。Sanon等(2012) 通过计算不同情景下生态系统服务在散点图中的位 置与两种生态系统服务同时达到最大值时所处位置 之间的距离作为两种生态系统服务权衡关系的度量 值。Lester等(2013)根据帕累托边界的形态, 将生态 系统服务权衡总结为6种(图4), 对每种权衡类型都 可以根据目前生态系统服务在帕累托图中所处的位 置, 确定生态系统服务当前状态是最优还是次优, 进而通过管理措施尽可能在不减少其中一种生态系 统服务供给量的同时, 增加其他生态系统服务的供 给量。采用约束线方法能够有效刻画生态系统服务 之间的限制作用，在此基础上，将约束线方法与帕 累托最优理论相结合, 能够为优化生态系统服务提 供一条新思路。

约束线方法具有解决优化问题的巨大潜力。土 地系统设计是适应并缓解气候变化的有效手段之 一, 已有研究表明地表覆盖对大气有反馈作用(Cao et al., 2015), 影响地气相互作用的因素有很多, 包 括地形、气候场、植被覆盖度、地表覆盖类型等, 采 用约束线方法能够全面理解这些因子间的相互作 用, 以及不同地气相互作用过程中的主要限制因子 与过程。同时, 在土地系统设计过程中, 社会经济发 展是必须要考虑的因素, 将约束线方法与经济学中 的优化问题(如帕累托效率)相结合, 综合考虑土地 利用/覆盖、气候变化以及经济发展, 为区域土地系 

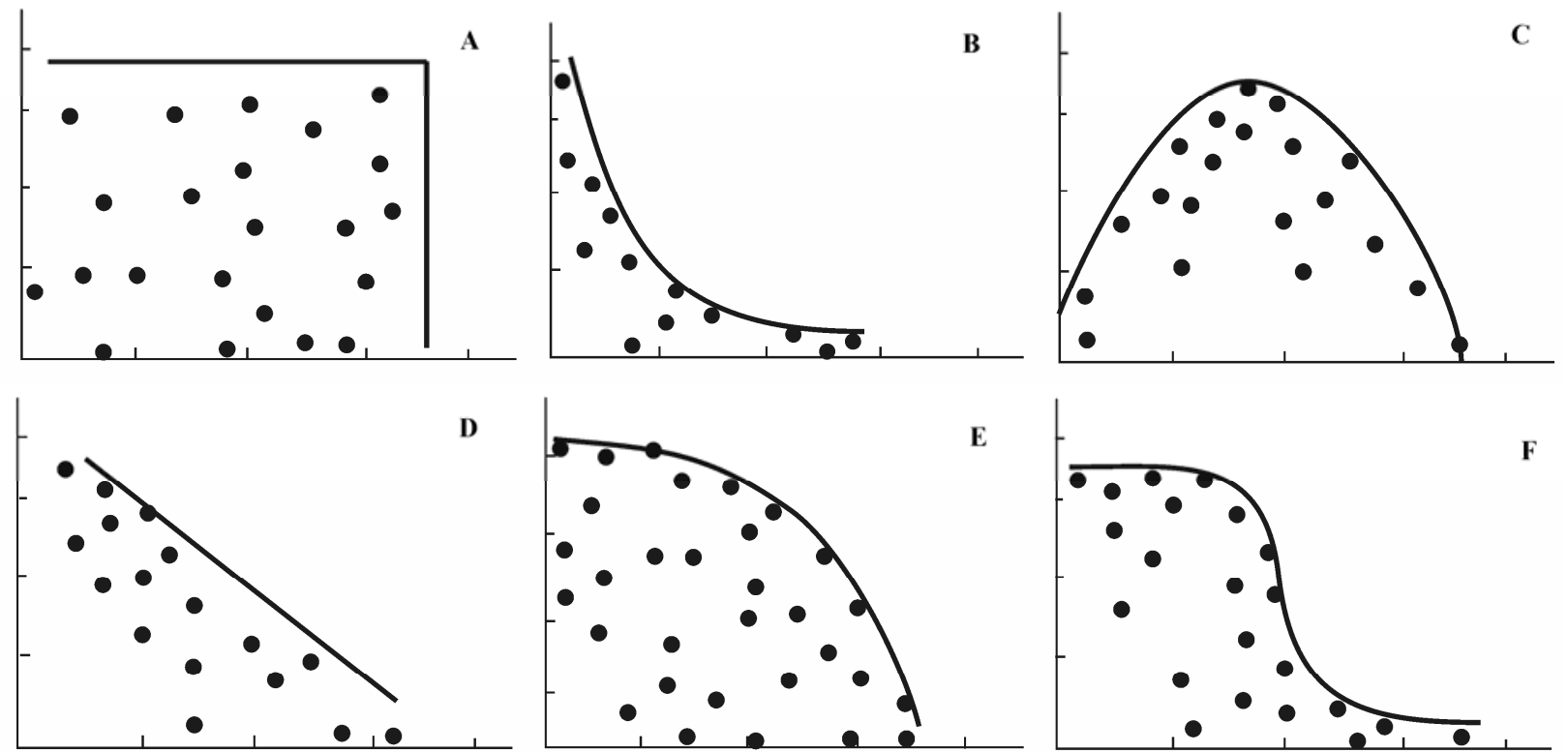

图4 生态系统服务关系示意图(横纵坐标分别代表两种生态系统服务, 修改自Lester等(2013))。

Fig. 4 Examples of some common types of relationships between ecosystem services (Modified from Lester et al. (2013)).

统优化提供有效建议，也是值得探索的方向。

综上所述，尽管存在一系列的问题与挑战，约 束线方法可以帮助研究者从散乱分布的数据云中提 取有效信息, 成为清晰理解变量之间限制作用的有 效手段。约束线所表征的变量分布范围和最大值, 能够广泛地为生态资源管理、资源优化配置以及实 现区域可持续发展提供重要的理论依据与技术支 撑。

\section{基金项目 国家自然科学基金(41571170)。}

致谢 本研究受到地表过程与资源生态国家重点实 验室项目(2015-ZY-18)资助，特此致谢。

\section{参考文献}

Alcamo J, Bennett EM, Alcamo J (2003). Ecosystems and human well-being: A framework for assessment. Island Press, 4(3-4), 199-213.

Anderson KJ, Jetz W (2005). The broad scale ecology of energy expenditure of endotherms. Ecology Letters, 8, 310-318.

Austin M (2007). Species distribution models and ecological theory: A critical assessment and some possible new approaches. Ecological Modelling, 200, 1-19.

Bennett EM, Peterson GD, Gordon LJ (2009). Understanding relationships among multiple ecosystem services. Ecology Letters, 12, 1394-1404.

Blackburn TM, Lawton JH, Perry JN (1992). A method of estimating the slope of upper bounds of plots of body size and abundance in natural animal assemblages. Oikos,
107-112.

Cade BS, Guo Q (2000). Estimating effects of constraints on plant performance with regression quantiles. Oikos, 245-254.

Cade BS, Noon BR (2003). A gentle introduction to quantile regression for ecologists. Frontiers in Ecology and the Environment, 1, 412-420.

Cade BS, Terrell JW, Schroeder RL (1999). Estimating effects of limiting factors with regression quantiles. Ecology, 80, 311-323.

Cao Q, Yu D, Georgescu M, Han Z, Wu J (2015). Impacts of land use and land cover change on regional climate: A case study in the agro-pastoral transitional zone of china. Environmental Research Letters, 10, 124025.

Chassot E, Bonhommeau S, Dulvy NK, Mélin F, Watson R, Gascuel D, Le Pape O (2010). Global marine primary production constrains fisheries catches. Ecology Letters, 13, 495-505.

Clark DA, Clark DB (1999). Assessing the growth of tropical rain forest trees: Issues for forest modeling and management. Ecological Applications, 9, 981-997.

Coomes DA, Allen RB (2007). Effects of size, competition and altitude on tree growth. Journal of Ecology, 95, 10841097.

Evanylo G, Sumner M (1987). Utilization of the boundary line approach in the development of soil nutrient norms for soybean production 1. Communications in Soil Science \& Plant Analysis, 18, 1379-1401.

Guo Q, Brown JH, Enquist BJ (1998). Using constraint lines to characterize plant performance. Oikos, 83, 237-245.

Guo Q, Brown JH, Valone TJ, Kachman SD (2000). 
Constraints of seed size on plant distribution and abundance. Ecology, 81, 2149-2155.

Guo Q, Rundel PW (1998). Self-thinning in early post fire chaparral succession: Mechanisms, implications, and a combined approach. Ecology, 79, 579-586.

Horning M (2012). Constraint lines and performance envelopes in behavioral physiology: The case of the aerobic dive limit. Frontiers in Physiology, 3, 1-9.

Huston MA (1999). Local processes and regional patterns: Appropriate scales for understanding variation in the diversity of plants and animals. Oikos, 86, 393-401.

Jansen JM, Pronker AE, Kube S, Sokolowski A, Sola JC, Marquiegui MA, Schiedek D, Bonga SW, Wolowicz M, Hummel H (2007). Geographic and seasonal patterns and limits on the adaptive response to temperature of European Mytilus spp. and Macoma balthica populations. Oecologia, 154, 23-34.

Kaiser MS, Speckman PL, Jones JR (1994). Statistical models for limiting nutrient relations in inland waters. Journal of the American Statistical Association, 89, 410-423.

Krause Jensen D, Middelboe AL, Sand-Jensen K, Christensen PB (2000). Eelgrass, Zostera marina, growth along depth gradients: Upper boundaries of the variation as a powerful predictive tool. Oikos, 91, 233-244.

Lautenbach S, Volk M, Gruber B, Dormann CF, Strauch M, Seppelt R (2010). Quantifying Ecosystem Service TradeOffs. International Environmental Modelling and Software Society (iEMSs) 2010 International Congress on Environmental Modelling and Software Modelling for Environment's Sake, Fifth Biennial Meeting, Ottawa, Canada.

Lessin LM, Dyer AR, Goldberg DE (2001). Using upper boundary constraints to quantify competitive response of desert annuals. Oikos, 92, 153-159.

Lester SE, Costello C, Halpern BS, Gaines SD, White C, Barth JA (2013). Evaluating tradeoffs among ecosystem services to inform marine spatial planning. Marine Policy, 38, 80-89.

Medinski T (2007). Soil Chemical and Physical Properties and Their Influence on the Plant Species Richness of Arid South-West Africa. PhD dissertation, University of Stellenbosch, Bellville, South Africa.

Medinski T, Mills A, Esler K, Schmiedel U, Jürgens N (2010). Do soil properties constrain species richness? Insights from boundary line analysis across several biomes in south western Africa. Journal of Arid Environments, 74, 1052-1060.

Mills A, Fey M, Donaldson J, Todd S, Theron L (2009). Soil infiltrability as a driver of plant cover and species richness in the semi-arid Karoo, South Africa. Plant and Soil, 320, 321-332.

Nishihiro J, Washitani I, Thomson J, Thomson B (2000). Patterns and consequences of stigma height variation in a natural population of a distylous plant, Primula sieboldii. Functional Ecology, 14, 502-512.

Pittman S, Turnblom E (2003). A study of self-thinning using coupled allometric equations: Implications for coastal douglas-fir stand dynamics. Canadian Journal of Forest Research, 33, 1661-1669.

Raudsepp-Hearne C, Peterson GD, Bennett EM (2010). Ecosystem service bundles for analyzing tradeoffs in diverse landscapes. Proceedings of the National Academy of Sciences of the United States of America, 107, 5242-5247.

Roberts JH, Angermeier PL (2007). Spatiotemporal variability of stream habitat and movement of three species of fish. Oecologia, 151, 417-430.

Robertson BP, Gardner JP, Savage C (2015). Macrobenthic-mud relations strengthen the foundation for benthic index development: A case study from shallow, temperate New Zealand estuaries. Ecological Indicators, 58, 161-174.

Sanon S, Hein T, Douven W, Winkler P (2012). Quantifying ecosystem service trade-offs: The case of an urban floodplain in Vienna, Austria. Journal of Environmental Management, 111, 159-172.

Scharf FS, Juanes F, Sutherland M (1998). Inferring ecological relationships from the edges of scatter diagrams: Comparison of regression techniques. Ecology, 79, 448-460.

Schneider FD, Scheu S, Brose U (2012). Body mass constraints on feeding rates determine the consequences of predator loss. Ecology Letters, 15, 436-443.

Schnug E, Heym J, Achwan F (1996). Establishing critical values for soil and plant analysis by means of the boundary line development system (bolides). Communications in Soil Science \& Plant Analysis, 27, 2739-2748.

Schröder HK, Andersen HE, Kiehl K (2005). Rejecting the mean: Estimating the response of fen plant species to environmental factors by non-linear quantile regression. Journal of Vegetation Science, 16, 373-382.

Strong W (2011). Tree canopy effects on understory species abundance in high-latitude Populus tremuloides stands, Yukon, Canada. Community Ecology, 12, 89-98.

Sumner ME (1978). Interpretation of nutrient ratios in plant tissue. Communications in Soil Science and Plant Analysis, 9, 335-345.

Thomson JD, Weiblen G, Thomson BA, Alfaro S, Legendre P (1996). Untangling multiple factors in spatial distributions: Lilies, gophers, and rocks. Ecology, 77, 1698-1715.

Wairegi LW, van Asten PJ, Tenywa MM, Bekunda MA (2010). Abiotic constraints override biotic constraints in east African highland banana systems. Field Crops Research, 117, 146-153.

Walsh CJ, Roy AH, Feminella JW, Cottingham PD, Groffman

www.plant-ecology.com 
PM, Morgan RP (2005). The urban stream syndrome: Current knowledge and the search for a cure. Journal of the North American Benthological Society, 24, 706-723.

Wang L, Lyons J, Kanehl P, Bannerman R (2001). Impacts of urbanization on stream habitat and fish across multiple spatial scales. Environmental Management, 28, 255-266.

Wang N, Jassogne L, van Asten P, Mukasa D, Wanyama I, Kagezi G, Giller K (2015a). Evaluating coffee yield gaps and important biotic, abiotic, and management factors limiting coffee production in Uganda. European Journal of Agronomy, 63, 1-11.

Wang T, Feng L, Mou P, Wu J, Smith JL, Xiao W, Yang H,
Dou H, Zhao X, Cheng Y (2015b). Amur tigers and leopards returning to china: Direct evidence and a landscape conservation plan. Landscape Ecology, 1-13.

Webb R (1972). Use of the boundary line in the analysis of biological data. Journal of Horticultural Science, 47, 309-310.

Wu J (2013). Landscape sustainability science: Ecosystem services and human well-being in changing landscapes. Landscape Ecology, 28, 999-1023.

责任编委: 常 杰 责任编辑: 王 葳

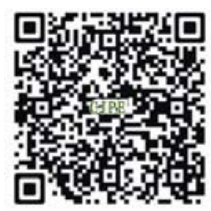

植物生态学报官网

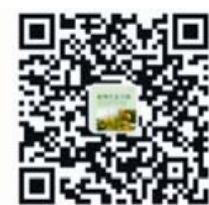

微信订阅号

期刊及学科

相关信息发布

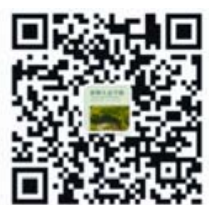

微信服务号

稿件状态查询

全文检索汶览 